\title{
ENTROPY AND ELLIPTIC EQUATIONS
}

\author{
BY \\ W. A. DAY \\ Hertford College, Oxford, England \\ In memory of my wife, Enid Patricia Day, who died 22 March 1991, aged 46 years
}

1. Introduction. Fourier's treatise on heat conduction [1] and Carnot's treatise on heat engines [2] were published within two years of each other-in 1822 and 1824, respectively - but there was little overlap between thermodynamics and the theory of heat conduction until recent times. Illuminating comments on this state of affairs are to be found in Truesdell's history of thermodynamics [3, pp. 47-137].

In [4] I proved two theorems about periodic solutions of parabolic equations that were suggested by considerations drawn from thermodynamics. The present paper aims to continue this line of investigation by proving new results about linear elliptic equations. These results, which depend on the positivity of certain kernels or Green's functions, involve the thermodynamic notion of entropy.

In order to motivate what we shall do, let us consider a homogeneous and isotropic rigid heat conductor that occupies a domain $\Omega \subset R^{3}$. If we suppose, for simplicity, that the thermal diffusivity is equal to unity then the temperature $u(x, t)$, at the point $x=\left(x_{1}, x_{2}, x_{3}\right) \in \Omega$ and time $t \in R$, satisfies the differential equation

$$
u_{t}=\Delta u+f(x) \text {, }
$$

where

$$
\Delta=\frac{\partial^{2}}{\partial x_{1}^{2}}+\frac{\partial^{2}}{\partial x_{2}^{2}}+\frac{\partial^{2}}{\partial x_{3}^{2}}
$$

is the Laplace operator and $f(x)$ is the heat supply to unit volume in unit time. Electrical heating, chemical reactions, radioactive decay, and the absorption of radiation may all contribute to a nonzero heat supply. We shall regard $f(x)$ as being at our disposal, subject to certain restrictions to be stated below.

If $u$ is positive, as will be the case if $u$ is measured on an absolute scale, we may divide Eq. (1.1) through by $u$ and obtain the equation

$$
\frac{u_{t}}{u}+\operatorname{div}\left(\frac{q}{u}\right)=\frac{|q|^{2}}{u^{2}}+\frac{f}{u},
$$

in which $q=-\operatorname{grad} u$ is the heat flux vector. Thus if $P \subset \Omega$ is a bounded subdomain and $\nu$ is the outward unit normal to the boundary $\partial P$, then on integrating both sides 
of (1.2) over $P$ and appealing to the divergence theorem, we arrive at the identity

$$
\frac{d}{d t} \int_{P} \log u d x+\int_{\partial P} \frac{\nu \cdot q}{u} d S=\int_{P} \frac{|q|^{2}}{u^{2}} d x+\int_{P} \frac{f}{u} d x
$$

where $d S$ is the element of surface area on $\partial P$ and $d x$ is the element of volume.

The terms that occur in the identity (1.3) have the following interpretations:

$\frac{d}{d t} \int_{P} \log u d x=$ rate of increase of entropy in $P$,

$\int_{\partial P} \frac{\nu \cdot q}{u} d S=$ flux of entropy out of $P$ due to heat conduction across $\partial P$,

$\int_{P} \frac{|q|^{2}}{u^{2}} d x=$ rate of generation of entropy within $P$ due

to internal heat conduction, $(1.5)$

$\int_{P} \frac{f}{u} d x=$ flux of entropy into $P$ due to the heat supply $f$.

It should be noted that the inequality

$$
\frac{d}{d t} \int_{P} \log u d x+\int_{\partial P} \frac{\nu \cdot q}{u} d x \geq \int_{P} \frac{f}{u} d S
$$

is an immediate consequence of (1.2). This last is the form that the Clausius-Duhem inequality takes in the present circumstances. One important modern approach to continuum thermodynamics starts by replacing the verbal statements of the second law that were proposed by Clausius and Kelvin with the Clausius-Duhem inequality. (For the work of Clausius and Kelvin, see, e.g., [3] and items 6, 7, 8 in the collection [5]. For the Clausius-Duhem inequality, see Coleman and Noll [6].)

In Secs. 2 and 3 we shall study the steady-state version of Eq. (1.1), which is just Poisson's equation, and ask how large the integrals (1.4), (1.5), and (1.6) can be made by varying the heat supply $f$ within certain constraints. In Sec. 4 we shall consider a more general class of linear elliptic equations that describe steady-state heat conduction in inhomogeneous and anisotropic rigid conductors.

2. Poisson's equation in $R^{3}$. We start by considering what happens when $\Omega=R^{3}$. We suppose that we are given a nonempty bounded domain $D \subset R^{3}$, and we require the heat supply $f$ to meet the following conditions:

$$
\begin{gathered}
f(x) \geq 0 \text { for } x \in R^{3}, \\
\text { supp } f \subset D, \\
f \in C^{\alpha}, \text { with a Hölder exponent in } 0<\alpha<1 .
\end{gathered}
$$

These conditions place no restriction upon the size of the integral $\int_{D} f d x$, which can be made arbitrarily large.

The collection of all heat supplies that satisfy (2.1), (2.2), and (2.3) will be denoted by $\mathscr{C}$; this collection is a cone, that is to say,

$$
\lambda f+\mu g \in \mathscr{C} \text { for } f, g \in \mathscr{C} \text { and } \lambda, \mu \geq 0 .
$$


The steady-state temperature $u(x)$ must now satisfy Poisson's equation

$$
\Delta u=-f(x) \text { for } x \in R^{3} \text {, }
$$

and we shall impose the condition

$$
u(x) \rightarrow u_{0} \quad \text { as }|x| \rightarrow \infty
$$

$u_{0}$ being a positive constant.

The unique solution of the problem consisting of (2.5) and (2.6) is

$$
u(x)=u_{0}+\frac{1}{4 \pi} \int_{D} \frac{f(y)}{|x-y|} d y
$$

and, necessarily, $u(x) \geq u_{0}>0$ for $x \in R^{3}$. Thus it is permissible to divide both sides of Eq. (2.5) by $u$ and to deduce, in the same way as we deduced (1.3), an identity

$$
\int_{\partial P} \frac{\nu \cdot q}{u} d S=\int_{P} \frac{|q|^{2}}{u^{2}} d x+\int_{P} \frac{f}{u} d x
$$

which holds if $P$ is a bounded domain.

With (2.8) in mind, we set

$$
F=\int_{R^{3}} \frac{f}{u} d x, \quad G=\int_{R^{3}} \frac{|q|^{2}}{u^{2}} d x, \quad H=G+F,
$$

the integrals being extended over all of $R^{3}$, and we make the interpretations

$F=$ flux of entropy into $R^{3}$ due to the heat supply $f$,

$G=$ rate of generation of entropy within $R^{3}$ due to internal heat conduction,

$H=$ flux of entropy out of $R^{3}$ due to heat conduction at infinity.

Lemma 1. If the heat supply $f \in \mathscr{C}$ then $F, G$, and $H$ are well defined and nonnegative; moreover,

$$
H=\frac{1}{u_{0}} \int_{D} f d x
$$

Proof. In order to prove the lemma we start with the observation that $F=$ $\int_{R^{3}} \frac{f}{u} d x=\int_{D} \frac{f}{u} d x$, from which it is clear that $F$ is well defined and nonnegative.

The monotone convergence theorem of integration theory assures us that if the limit

$$
\lim _{a \rightarrow \infty} \int_{B_{a}} \frac{|q|^{2}}{u^{2}} d x
$$

exists, where $B_{a}$ is the ball $|x| \leq a$, then $G$ is well defined as a Lebesgue integral and nonnegative. If, in fact, we can show that the limit exists and has the value

$$
\frac{1}{u_{0}} \int_{D} f d x-\int_{D} \frac{f}{u} d x
$$

we shall have completed the proof of the lemma, for then $H$ will be well defined by (2.9), it will be nonnegative, and its value will be as asserted in (2.10). 
With no loss of generality we may suppose that $0 \in D$ and that $D \subset B_{a}$. On setting $P=B_{a}$ in $(2.8)$, we see that

$$
\int_{B_{a}} \frac{|q|^{2}}{u^{2}} d x=\int_{\partial B_{a}} \frac{\nu \cdot q}{u} d S-\int_{D} \frac{f}{u} d x .
$$

The next step is to verify that

$$
\nu(x) \cdot q(x) \geq 0 \text { for } x \in \partial B_{a} .
$$

To do so we observe that, by virtue of (2.7),

$$
q(x)=-\operatorname{grad} u(x)=\frac{1}{4 \pi} \int_{D} \frac{f(y)}{|x-y|^{3}}(x-y) d y .
$$

If $x \in \partial B_{a}$ then $\nu(x) \cdot x=a$, and so

$$
\begin{aligned}
\nu(x) \cdot q(x) & =\frac{1}{4 \pi} \int_{D} \frac{f(y)}{|x-y|^{3}} \nu(x) \cdot(x-y) d y \\
& =\frac{1}{4 \pi} \int_{D} \frac{f(y)}{|x-y|^{3}}(a-\nu(x) \cdot y) d y \\
& \geq \frac{1}{4 \pi} \int_{D} \frac{f(y)}{|x-y|^{3}}(a-|y|) d y \geq 0,
\end{aligned}
$$

as was asserted to be the case.

Now let $U(a)=\max \{u(x):|x|=a\}$. Then $U(a) \geq u_{0}$ and, by virtue of the condition (2.6), $U(a) \rightarrow u_{0}$ as $a \rightarrow \infty$. Thus, since (2.14) holds, it follows that

$$
\frac{1}{U(a)} \int_{\partial B_{a}} \nu \cdot q d S \leq \int_{\partial B_{a}} \frac{\nu \cdot q}{u} d S \leq \frac{1}{u_{0}} \int_{\partial B_{a}} \nu \cdot q d S .
$$

However, Poisson's equation (2.5) implies that

$$
\begin{aligned}
\int_{\partial B_{a}} \nu \cdot q d S & =-\int_{\partial B_{a}} \nu \cdot \operatorname{grad} u d S \\
& =-\int_{B_{a}} \Delta u d x=\int_{B_{a}} f d x=\int_{D} f d x .
\end{aligned}
$$

Hence,

$$
\frac{1}{U(a)} \int_{D} f d x \leq \int_{\partial B_{a}} \frac{\nu \cdot q}{u} d S \leq \frac{1}{u_{0}} \int_{D} f d x
$$

and, on letting $a \rightarrow \infty$, we see that

$$
\lim _{a \rightarrow \infty} \int_{\partial B_{a}} \frac{\nu \cdot q}{u} d S=\frac{1}{u_{0}} \int_{D} f d x .
$$

Thus, on letting $a \rightarrow \infty$ in (2.13), we conclude that the limit (2.11) exists and has the value (2.12), which completes the proof of Lemma 1 .

Lemma 1 permits us to think of $F, G$, and $H$ as nonnegative functionals defined on the cone $\mathscr{C}$, and it is clear from the formula (2.10) that $H$ has the property

$$
H[\lambda f+\mu g]=\lambda H[f]+\mu H[g] \text { for } f, g \in \mathscr{C} \text { and } \lambda, \mu \geq 0 .
$$

However, $F$ and $G$ do not have this property. 
The chief result of this section is

TheOREM 1. Neither $G$ nor $H$ is bounded above on $\mathscr{C}$, but

$$
F[f] \leq 4 \pi \operatorname{diam}(D) \text { for } f \in \mathscr{C} .
$$

Thus, by controlling the heat supply $f$, we can make the rate of generation of entropy within $R^{3}$ as large as we like, and the same is true of the flux of entropy out of $R^{3}$ due to heat conduction at infinity. By contrast, though, we cannot make the flux of entropy into $R^{3}$ due to the heat supply as large as we like in the circumstances envisaged. We conclude that whenever there is a large flux of entropy out of $R^{3}$ due to heat conduction at infinity, the bulk of that entropy is not entropy directly associated with the heat supply that we control, even though the heat supply be large, but is entropy generated indirectly by the mechanism of internal heat conduction.

We turn now to the proof of Theorem 1 . That $H$ is not bounded above follows immediately from formula (2.10). If we can prove estimate (2.16), it will then follow from (2.9) that $G$ is not bounded above. In order to prove (2.16), we need only note that

$$
F[f]=\int_{D} \frac{f(x)}{u(x)} d x
$$

while the representation (2.7) tells us that, for $x \in D$,

$$
u(x) \geq \frac{1}{4 \pi \operatorname{diam}(D)} \int_{D} f(y) d y .
$$

Hence

$$
F[f] \leq 4 \pi \operatorname{diam}(D) \int_{D}\left(\int_{D} f(y) d y\right)^{-1} f(x) d x=4 \pi \operatorname{diam}(D),
$$

which is (2.16), and the proof is complete.

3. Poisson's equation in a bounded domain. We suppose now that $\Omega \subset R^{3}$ is a bounded $C^{2, \alpha}$ domain and that $D$ is a nonempty domain contained strictly within $\Omega$, that is to say, $D \subset \Omega$ and $\operatorname{dist}(D, \partial \Omega)>0$. The heat supply $f$ is required to meet the conditions:

$$
\begin{aligned}
& f(x) \geq 0 \text { for } x \in \bar{\Omega}, \\
& \operatorname{supp} f \subset D, \\
& f \in C^{\alpha}(\bar{\Omega}),
\end{aligned}
$$

and once again the collection $\mathscr{E}$, consisting of all such heat supplies, is a cone.

As before, the steady-state temperature $u(x)$ satisfies Poisson's equation

$$
\Delta u=-f(x) \text { for } x \in \Omega .
$$

We impose the boundary condition

$$
u(x)=u_{0} \text { for } x \in \partial \Omega,
$$

$u_{0}$ being a positive constant. As is well known (Gilbarg and Trudinger [7, Theorem 6.14]) this Dirichlet problem has a unique solution $u \in C^{2, \alpha}(\bar{\Omega})$. 
The maximum principle guarantees that $u(x) \geq u_{0}>0$ for $x \in \bar{\Omega}$. Thus it is permissible to divide Poisson's equation through by $u$ to obtain the identity

$$
\operatorname{div}\left(\frac{q}{u}\right)=\frac{|q|^{2}}{u^{2}}+\frac{f}{u}
$$

and, on integrating both sides over $\Omega$ and using the divergence theorem, we deduce the equation

$$
H=G+F
$$

in which

$$
\begin{aligned}
& F=\int_{\Omega} \frac{f}{u} d x=\text { flux of entropy into } \Omega \text { due to the heat supply } f \\
& G=\int_{\Omega} \frac{|q|^{2}}{u^{2}} d x=\text { rate of generation of entropy within } \Omega \\
& \quad \text { due to internal heat conduction, } \\
& H=\int_{\partial \Omega} \frac{\nu \cdot q}{u} d S=\text { flux of entropy out of } \Omega \text { due to heat conduction across } \partial \Omega .
\end{aligned}
$$

The following counterpart to Lemma 1 is now valid.

LemMa 2. If $f \in \mathscr{C}$ then $F, G$, and $H$ are nonnegative and

$$
H=\frac{1}{u_{0}} \int_{D} f d x .
$$

Proof. It is clear from the definition of $F$ and the restrictions $f \geq 0$ and $u>0$, that $F \geq 0$. Likewise the definition of $G$ tells us immediately that $G \geq 0$ and hence, we have $H=G+F \geq 0$. Moreover, if we invoke Poisson's equation and the boundary condition on $u$, we see that

$$
\begin{aligned}
H & =\int_{\partial \Omega} \frac{\nu \cdot q}{u} S=\frac{1}{u_{0}} \int_{\partial \Omega} \nu \cdot q d S \\
& =-\frac{1}{u_{0}} \int_{\partial \Omega} \nu \cdot \operatorname{grad} u d s=-\frac{1}{u_{0}} \int_{\Omega} \Delta u d x \\
& =\frac{1}{u_{0}} \int_{\Omega} f d x=\frac{1}{u_{0}} \int_{D} f d x,
\end{aligned}
$$

which proves the lemma.

As we did before, we think of $F, G$, and $H$ as functionals defined on the cone $\mathscr{C}$. In view of (3.4), $H$ has property (2.15).

Theorem 2. (i) Neither $G$ nor $H$ is bounded above on $\mathscr{C}$, but there is a positive constant $M$ depending only upon the domains $\Omega$ and $D$ such that

$$
F[f] \leq M \quad \text { for } f \in \mathscr{C} .
$$

(ii) If $\Omega$ is a ball of volume $|\Omega|$ we can take

$$
M=\frac{30 \cdot 5^{1 / 2} \cdot|\Omega|}{[\operatorname{dist}(D, \partial \Omega)]^{2}} .
$$


Part (i) of Theorem 2 shows that what was asserted in Theorem 1 when $\Omega=R^{3}$ remains true when $\Omega$ is a bounded domain. That is to say, by controlling the heat supply $f$, we can make the rate of generation of entropy within $\Omega$ as large as we like, and the same is true of the flux of entropy out of $\Omega$ due to heat conduction across $\partial \Omega$. However, the flux of entropy into $\Omega$ due to the heat supply cannot be made arbitrarily large.

The bound (3.6) has the property that $M \rightarrow \infty$ when $\operatorname{dist}(D, \partial \Omega) \rightarrow 0$, a fact that suggests that $F$ may be unbounded if the requirement that $\operatorname{dist}(D, \partial \Omega)>0$ is relaxed. This can be seen to be so by considering what happens when $\Omega$ is the ball $|x|<a, D=\Omega$, and $f(x) \equiv \lambda$ where $\lambda$ is a large positive constant. In these circumstances the unique solution of the Dirichlet problem

$$
\begin{array}{ccrl}
\Delta u=-\lambda & & \text { in }|x|<a, \\
u(x)=u_{0} & & \text { when }|x|=a
\end{array}
$$

is

$$
u(x)=u_{0}+\frac{\lambda}{6}\left(a^{2}-|x|^{2}\right)
$$

therefore,

$$
\begin{aligned}
F & =\int_{\Omega} \frac{\lambda}{u} d x=\int_{0}^{a} \frac{4 \pi \lambda r^{2} d r}{u_{0}+(\lambda / 6)\left(a^{2}-r^{2}\right)} \\
& =12 \pi b \log \left(\frac{b+a}{b-a}\right)-24 \pi a
\end{aligned}
$$

where $b=\left(a^{2}+6 u_{0} / \lambda\right)^{1 / 2}$. On letting $\lambda \rightarrow \infty$ we see that $b \rightarrow a$ and, therefore, $F \rightarrow \infty$. Hence $F$ cannot be bounded on $\mathscr{C}$.

We turn to the proof of Theorem 2. It is clear from (3.4) that $H$ is not bounded above on $\mathscr{C}$, and once we have proved that $F$ is bounded above on $\mathscr{C}$ it will then follow from (3.3) that $G$ is not bounded above on $\mathscr{C}$.

In order to prove that $F$ is bounded above, we appeal to the fact that the solution of the Dirichlet problem that consists of (3.1) and (3.2) admits the representation

$$
u(x)=u_{0}+\int_{\Omega} K(x, y) f(y) d y,
$$

in which the kernel $K(x, y)$ is strictly positive and continuous for $x, y \in \Omega$ and $x \neq y$. Hence, if $f \in \mathscr{C}$ then

$$
\begin{aligned}
F & =\int_{\Omega} \frac{f}{u} d x=\int_{D} \frac{f}{u} d x \\
& =\int_{D}\left(u_{0}+\int_{D} K(x, y) f(y) d y\right)^{-1} f(x) d x \\
& \leq m^{-1} \int_{D}\left(\int_{D} f(y) d y\right)^{-1} f(x) d x=m^{-1}
\end{aligned}
$$

where

$$
m=\text { g. l.b. }\{K(x, y): x, y \in D\}>0,
$$


which confirms (3.5), with $M=m^{-1}$. As the theorem claims, $M$ depends only on the domains $\Omega$ and $D$, and so the proof of part (i) is complete.

In order to prove part (ii), let $\Omega$ be the ball $|x|<a$. It will be recalled that the associated kernel is then

$$
K(x, y)=\left\{\begin{array}{l}
\frac{1}{4 \pi}\left[\frac{1}{|x-y|}-\frac{a}{\left.|x|\left|y-a^{2} x /\right| x\right|^{2} \mid}\right] \text { for } x \neq 0, \\
\frac{1}{4 \pi}\left[\frac{1}{|y|}-\frac{1}{a}\right] \text { for } x=0,
\end{array}\right.
$$

but, for our purpose, this is not the most convenient form in which to express the kernel. If, though, we appeal to the identity

$$
\frac{|x|^{2}}{a^{2}}\left|y-\frac{a^{2}}{|x|^{2}} x\right|^{2}=|x-y|^{2}+\frac{1}{a^{2}}\left(a^{2}-|x|^{2}\right)\left(a^{2}-|y|^{2}\right)
$$

and put

$$
\phi=|x-y|^{2}, \quad \psi=\frac{1}{a^{2}}\left(a^{2}-|x|^{2}\right)\left(a^{2}-|y|^{2}\right),
$$

we arrive at the alternative expression

$$
K(x, y)=\frac{1}{4 \pi}\left(\frac{1}{\phi^{1 / 2}}-\frac{1}{(\phi+\psi)^{1 / 2}}\right) .
$$

It follows from the definitions of $\phi$ and $\psi$ that if $x, y \in D$ then

$$
\begin{aligned}
\psi & =\frac{1}{a^{2}}(a-|x|)(a+|x|)(a-|y|)(a+|y|) \\
& \geq(a-|x|)(a-|y|) \geq[\operatorname{dist}(D, \partial \Omega)]^{2}
\end{aligned}
$$

and

$$
\phi \leq 4 a^{2}, \quad \psi \leq a^{2} .
$$

On combining (3.7) with the estimates (3.8), (3.9), and the elementary inequality

$$
\frac{1}{\phi^{1 / 2}}-\frac{1}{(\phi+\psi)^{1 / 2}}=\frac{1}{2} \int_{\phi}^{\phi+\psi} \frac{d \sigma}{\sigma^{3 / 2}} \geq \frac{\psi}{2(\phi+\psi)^{1 / 2}} \quad \text { for } \phi, \psi>0,
$$

we see that for $x, y \in D$,

$$
K(x, y) \geq \frac{[\operatorname{dist}(D, \partial \Omega)]^{2}}{8 \pi\left(5 a^{2}\right)^{3 / 2}}=\frac{[\operatorname{dist}(D, \partial \Omega)]^{2}}{30 \cdot 5^{1 / 2} \cdot|\Omega|}
$$

which shows that $M$ can be taken to have the value (3.6). Thus the proof of Theorem 2 is complete.

4. Generalisation to other elliptic equations. Theorem 2 can be generalised without difficulty to other elliptic equations in arbitrarily many independent variables. Thus let $\Omega \subset R^{n} \quad(n \geq 2)$ be a bounded $C^{2, \alpha}$ domain and let $D$ be a nonempty domain contained strictly within $\Omega$. We consider the Dirichlet problem

$$
\begin{aligned}
L u & =-f(x) & & \text { for } x \in \Omega \\
u(x) & =u_{0} & & \text { for } x \in \partial \Omega,
\end{aligned}
$$


in which the differential operator is

$$
L=\sum_{i, j=1}^{n} \frac{\partial}{\partial x_{i}}\left(a_{i j}(x) \frac{\partial}{\partial x_{j}}\right)
$$

and $u_{0}$ is a positive constant. We require $f$ to belong to the collection $\mathscr{C}$, consisting of all heat supplies that meet the same conditions as in Sec. 3.

The coefficients $a_{i j}(x)$, which are thermal conductivities when $n=3$, are restricted by requiring that they be in $C^{1, \alpha}(\bar{\Omega})$ and that the operator $L$ be strictly elliptic, that is to say, we suppose there to be a positive constant $\lambda$ such that

$$
\sum_{i, j=1}^{n} a_{i j}(x) \xi_{i} \xi_{j} \geq \lambda \sum_{i=1}^{n} \xi_{i}^{2}
$$

for every $x=\left(x_{1}, \ldots, x_{n}\right) \in \Omega$ and every $\xi=\left(\xi_{1}, \ldots, \xi_{n}\right)$. In these circumstances the Dirichlet problem has a unique solution $u \in C^{2, \alpha}(\bar{\Omega})$.

In view of the weak maximum principle and the restrictions of sign imposed upon $f$ and $u_{0}$, it must be that $u(x) \geq u_{0}>0$ for $x \in \bar{\Omega}$. Thus we can divide the differential equation through by $u$ and, on doing so, we can deduce an identity

$$
H=G+F \text {, }
$$

in which

$$
\begin{aligned}
F & =\int_{\Omega} \frac{f}{u} d x \\
G & =\int_{\Omega} \frac{1}{u^{2}} \sum_{i, j=1}^{n} a_{i j} \frac{\partial u}{\partial x_{i}} \frac{\partial u}{\partial x_{j}} d x \\
H & =\frac{1}{u_{0}} \int_{\Omega} f d x
\end{aligned}
$$

It is clear that $F$ is nonnegative. Moreover, the strict ellipticity of $L$ guarantees that $G$ is nonnegative and, hence, $H$ too must be nonnegative. The generalised version of Theorem 2 is

TheOREM 3. (i) Neither $G$ nor $H$ is bounded above on $\mathscr{C}$, but there is a positive constant $M$ depending only upon the domains $\Omega$ and $D$ and the operator $L$ such that

$$
F[f] \leq M \quad \text { for } f \in \mathscr{C}
$$

(ii) If $L$ is the Laplacian

$$
\frac{\partial^{2}}{\partial x_{1}^{2}}+\cdots+\frac{\partial^{2}}{\partial x_{n}^{2}}
$$

and $\Omega$ is a ball of hypervolume $|\Omega|$ then we can take

$$
M=\frac{2 n \cdot 5^{n / 2} \cdot|\Omega|}{[\operatorname{dist}(D, \partial \Omega)]^{2}} .
$$

That $H$ is not bounded above follows immediately from (4.2), and once we have shown that (4.3) holds it will follow immediately from (4.1) that $G$ is not bounded 
above. In order to prove (4.3) we proceed exactly as before, that is to say, we appeal to the fact that the solution of the Dirichlet problem admits the representation (Miranda $[8$, Theorem 21, VI])

$$
u(x)=u_{0}+\int_{\Omega} K(x, y) f(y) d y
$$

in which the kernel $K(x, y)$ is strictly positive and continuous for $x, y \in \Omega$ and $x \neq y$. Hence (4.3) holds with $M=m^{-1}$, where

$$
m=\text { g. l.b. }\{K(x, y): x, y \in D\}>0 .
$$

When $L$ is the Laplacian and $\Omega$ is a ball, we can construct the kernel explicitly and deduce (4.4) by using essentially the same argument as before; when $n=2$ the argument requires slight modification to take account of the logarithmic singularity of the kernel.

\section{REFERENCES}

[1] J. Fourier, Théorie Analytique de la Chaleur, Paris, 1822

[2] S. Carnot, Réflexions sur la Puissance Motrice du Feu et sur les Machines Propres à Développer cette Puissance, Paris, 1824

[3] C. Truesdell, The Tragicomical History of Thermodynamics 1822-1854, Springer-Verlag, New York, 1980

[4] W. A. Day, Parabolic equations and thermodynamics, Quart. Appl. Math. 50, 523-533 (1992)

[5] J. Kestin, ed., The Second Law of Thermodynamics, Benchmark papers on Energy/5, Dowden, Hutchinson, and Ross, Stroudsburg, PA, 1976

[6] B. D. Coleman and W. Noll, The thermodynamics of elastic materials with heat conduction and viscosity, Arch. Rational Mech. Anal. 13, 167-178 (1963)

[7] D. Gilbarg and N. S. Trudinger, Elliptic Partial Differential Equations of Second Order, SpringerVerlag, Berlin, 1977

[8] C. Miranda, Partial Differential Equations of Elliptic Type, 2nd revised ed., Springer-Verlag, Berlin, 1970 